\title{
In-situ TEM on Formation of Monatomic Metallic Glasses Through Ultrafast Liquid Quenching
}

\author{
Scott X. Mao ${ }^{1 *}$ \\ 1. Department of Mechanical Engineering and Materials Science, University of Pittsburgh, \\ Pittsburgh, PA, USA. \\ *Corresponding author: sxm2@pitt.edu
}

Metallic glasses, (MGs) as a materials class with peculiar properties, are normally prepared by quenching from metaling states with high cooling rate. It has long been supposed that any metallic liquid can be transformed into a glassy state if the cooling rate is sufficiently high [1,2]. Numerous MGs consisting of at least two elements could be formed by various cooling techniques, but the vitrification of monatomic metallic glass has not been achieved under true laboratory conditions yet due to the poor glass-forming ability of elemental materials.[3] The monatomic glasses are easy to transform into crystalline states with high crystallization rate even at room temperature and the poor thermal stability makes it hard to investigate their detailed microstructures. Developing advanced techniques with extremely high cooling rate to fabricate monatomic metallics glasses and control the corresponding crystallization process is significant to investigate the inherent structure and kinetic behavior of liquids [4] and expand the cognition of MGs.

Here we report an experimental method to obtain monatomic metallic glasses by conducting a liquid quenching with an unprecedented high rate of $10^{14} \mathrm{~K} / \mathrm{s}$. [5] Figure 1 shows the technique of forming monatomic Mgs through ultrafast quenching. First, two clean nano-tips of monatomic metals contact with each other (Fig. 1a) under an ultrahigh vacuum condition inside the TEM. Then applying a short square electric pulse, typically 0.5-3 V in amplitude and within $3.7 \mathrm{~ns}$ in duration, generates local Joule heating on these two tips, making them melt together and form a melting zone in the middle (Fig. 1b). The high heat dissipating rate through solidifying melt piece and the neighboring substrates leads to the ultrahigh cooling rate to quench the liquid melt into solid glass. The MG of pure refractory body-centered cubic (bcc) Ta is firstly vitrified from liquid melts through this technique, as shown in d-e. Corresponding Fast Fourier transformation patterns (Figs. $1 \mathrm{f}-\mathrm{h}$ ) of the vitrified region and two crystalline substrates in (e) confirmed that the Ta MG is formed after melting and following rapid cooling. Monatomic metallic glasses of transition metals, $\mathrm{V}$ and $\mathrm{W}$, were successfully obtained by using this vitrification technique and the competition between the quenching rate and the crystallization rate determines the glass formability of metals. The reversible vitrification-crystallization processes are easy to be controlled by adjusting the electric pulse in our experiment. The Ta MG formed after first vitrification pulse and it reverted completely to the original crystalline after sixth crystallization pulse with low voltage. The reversible phase process could be achieved by reversing the vitrification-crystallization. Although monatomic metallic glasses are generally deemed to be unstable, the transition metal metallic glasses showed slow crystallization rate at room temperature without extra heating. These features make it possible to study the dynamic structure evolution between the glassy and crystal states under high resolution TEM observation. The stable monatomic metallic glasses obtained 
by our approach provide opportunities to study their structure-property relationship. Moreover, the transition between glassy and crystalline states achieved by the technique showed here brings out potential of monatomic metallics in micro-electro-mechanical applications like signal transmission. Results of single elemental face-centered cubic (FCC) and hexagonal close-packed (HCP) metallic liquids through this ultrafast liquid quenching will also be presented.[6]

\section{References:}

[1] Greer, A. Lindsay, Science 267 (1995), p. 1947.

[2] Turnbull, David, Phys. 10 (1969), p. 473.

[3] Broughton, J.Q., et al., Physical review letters 20 (1982), p.1496.

[4] Stillinger, F. H. and Weber, T. A, Science 225 (1984), p. 983.

[5] Zhong, L, et al., Nature 512 (2014), p. 177.

[6] The author acknowledges National Science Foundation (NSF) grant CMMI 0928517 through the support of the University of Pittsburgh.
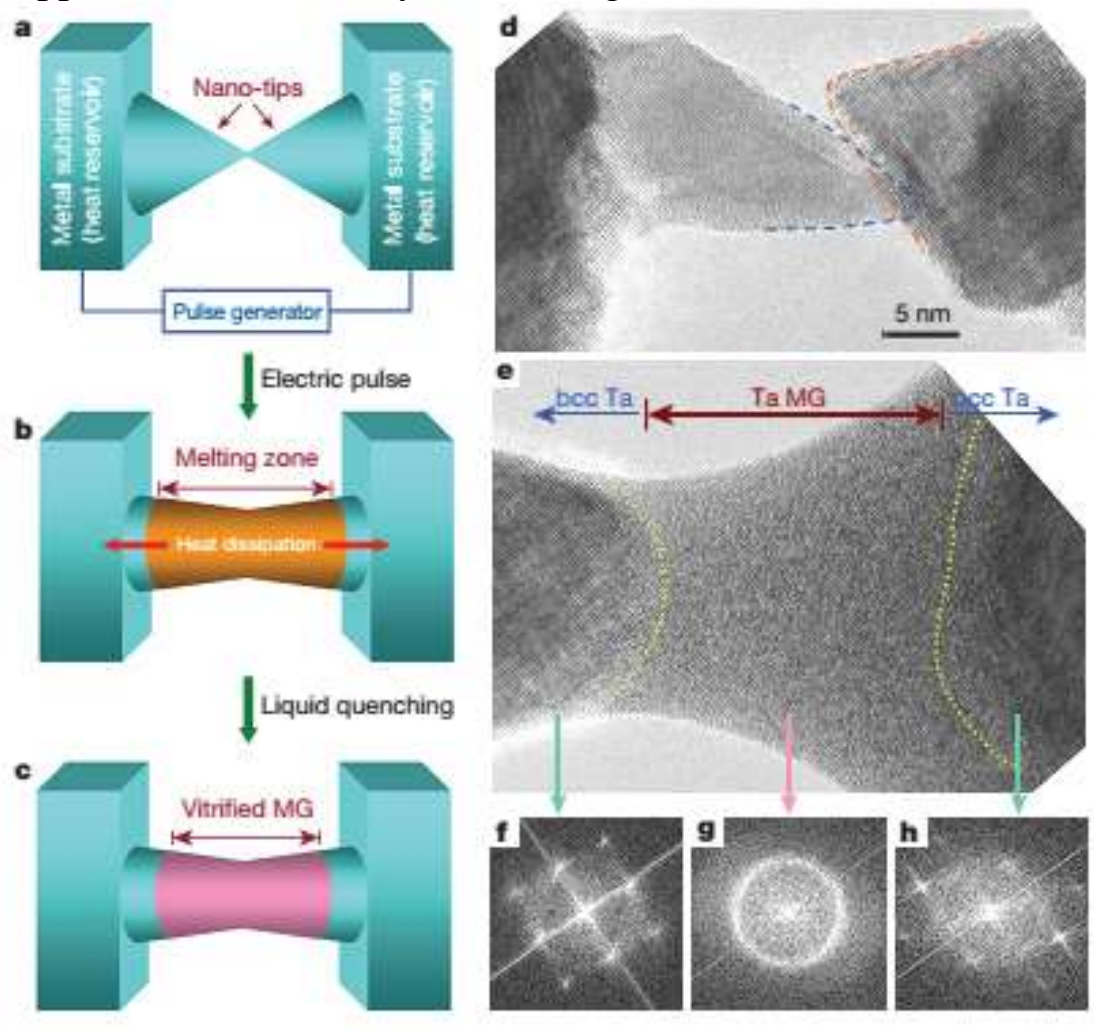

Figure 1. Illustration of an ultrafast liquid quenching approach. (a-c) Schematic drawing of the experimental configuration. Two contacting nano-tips (a) are melted together by applying a short electric pulse (b). Vitrified monatomic metallic glass is formed in the melting zone through rapidly dissipating heat to two neighboring substrates (c). d, e, Highresolution TEM images of two Ta nano-tips (d) formed Ta metallic glass (e) after a short electric pulse. (f-h) Corresponding Fast Fourier transformation patterns of the vitrified region and two crystalline substrates in (e). Reused by the author of the original work-Ref [5]. 